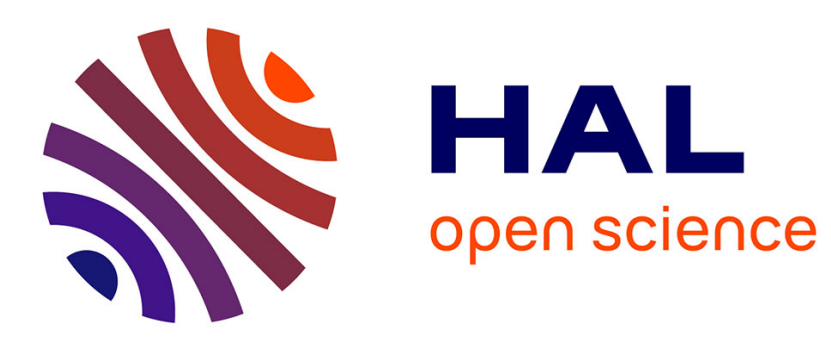

\title{
Mesures de conductivité par la méthode de perturbation en cavité hyperfréquence mesures en champ magnétique
}

\author{
J.C. Caëbrou, A. Coumes
}

\section{To cite this version:}

J.C. Caëbrou, A. Coumes. Mesures de conductivité par la méthode de perturbation en cavité hyperfréquence mesures en champ magnétique. Revue de Physique Appliquée, 1971, 6 (2), pp.217-221. 10.1051/rphysap:0197100602021700 . jpa-00243530

\section{HAL Id: jpa-00243530 https://hal.science/jpa-00243530}

Submitted on 1 Jan 1971

HAL is a multi-disciplinary open access archive for the deposit and dissemination of scientific research documents, whether they are published or not. The documents may come from teaching and research institutions in France or abroad, or from public or private research centers.
L'archive ouverte pluridisciplinaire HAL, est destinée au dépôt et à la diffusion de documents scientifiques de niveau recherche, publiés ou non, émanant des établissements d'enseignement et de recherche français ou étrangers, des laboratoires publics ou privés. 


\title{
MESURES DE CONDUCTIVITÉ PAR LA MÉTHODE DE PERTURBATION EN CAVITÉ HYPERFRÉQUENCE MESURES EN CHAMP MAGNÉTIQUE
}

\author{
J. C. CAËROU et A. COUMES
}

Laboratoire d'Electromagnétisme

E. N. S. d'Electronique et de Radioélectricité de Grenoble

1. Choix de la méthode. - Les mesures de conductivités et d'effet Hall se font habituellement par la méthode des quatre points. Toutefois, dans le cas des semi-conducteurs, cette méthode offre parfois de nombreux inconvénients tels que la taille d'un échantillon selon une forme compliquée, l'établissement de contacts ohmiques entre les fils d'amenée de courant et le semi-conducteur.

D'autre part, l'antimoniure d'indium (InSb) ayant une conduction élevée, il faudrait faire passer des courants intenses dans l'échantillon pour mesurer des chutes de potentiel notables aux bornes de ce dernier.

Ces quelques remarques suffisent pour chercher des moyens différents pour effectuer des mesures. Nous utiliserons donc des méthodes hyperfréquences sans contact.

En hyperfréquence, les méthodes consisteront à mesurer l'interaction des ondes et de la matière. Les hyperfréquences nous offrent de nombreuses possibilités ; seules les difficultés de calculs semblent limiter le choix. Nous pouvons distinguer :

1.1 LeS MESURES EN GUIDES D'ONDE [1], [2], [3], [4], [5]. - 1.1.1 Par réflexion.

1.1.2 Par transmission à travers une lame. - Les mesures en guides sont excellentes dans la plupart des cas, malheureusement, l'antimoniure d'indium étant très conducteur, les méthodes précédentes sont à la limite de leurs possibilités.

Il faut, pour mesurer cette conductivité, utiliser un système à haute sensibilité. Les cavités hyperfréquences caractérisées par un facteur de qualité $Q$ élevé possèdent cette dernière caractéristique.

1.2 Mesures en CAVITÉ HYPERFRÉQUENCE. - Elles sont faites par la mesure de la perturbation provoquée par l'introduction d'un petit échantillon dans une région de champ magnétique ou électrique maximal de la cavité. Cette perturbation se traduit par une variation $\delta \omega$ de la fréquence de résonance et une variation $\delta Q$ du facteur de qualité de la cavité. Il nous faut trouver une relation entre les valeurs observables $\delta \omega$ et $\delta Q$ et les paramètres $\sigma, \varepsilon_{r}$ de l'échantillon.

Nous avons placé une petite sphère dans une région de champ magnétique maximal de la cavité; cette dernière méthode est plus sensible dans notre cas, à cause de la grande valeur de $\sigma$, que celle consistant à placer l'échantillon dans une région de champ électrique - le champ magnétique est supposé uniforme au niveau de la sphère.

Le facteur de qualité est mesuré en présence de l'échantillon $(Q)$ puis en son absence $\left(Q_{0}\right)$. Les pertes dues au spécimen se traduisent par un facteur de qualité $Q_{s}$ défini par : $1 / Q_{s}=(1 / Q)-\left(1 / Q_{0}\right)$; pour les fréquences utilisées et pour le domaine de résistivité, les pertes sont dues en grande partie aux courants de Foucault confinés en surface de l'échantillon.

2. Pertes par courants de Foucault dans une sphère homogène, isotrope. - Plaçons une sphère dans un champ magnétique alternatif homogène de direction $\mathrm{Oz}$ (Fig. 1) : $B=B_{0} . \mathrm{e}^{j \omega t}$. Ce champ magnétique induit des courants de Foucault dans la sphère. Ceux-ci sont

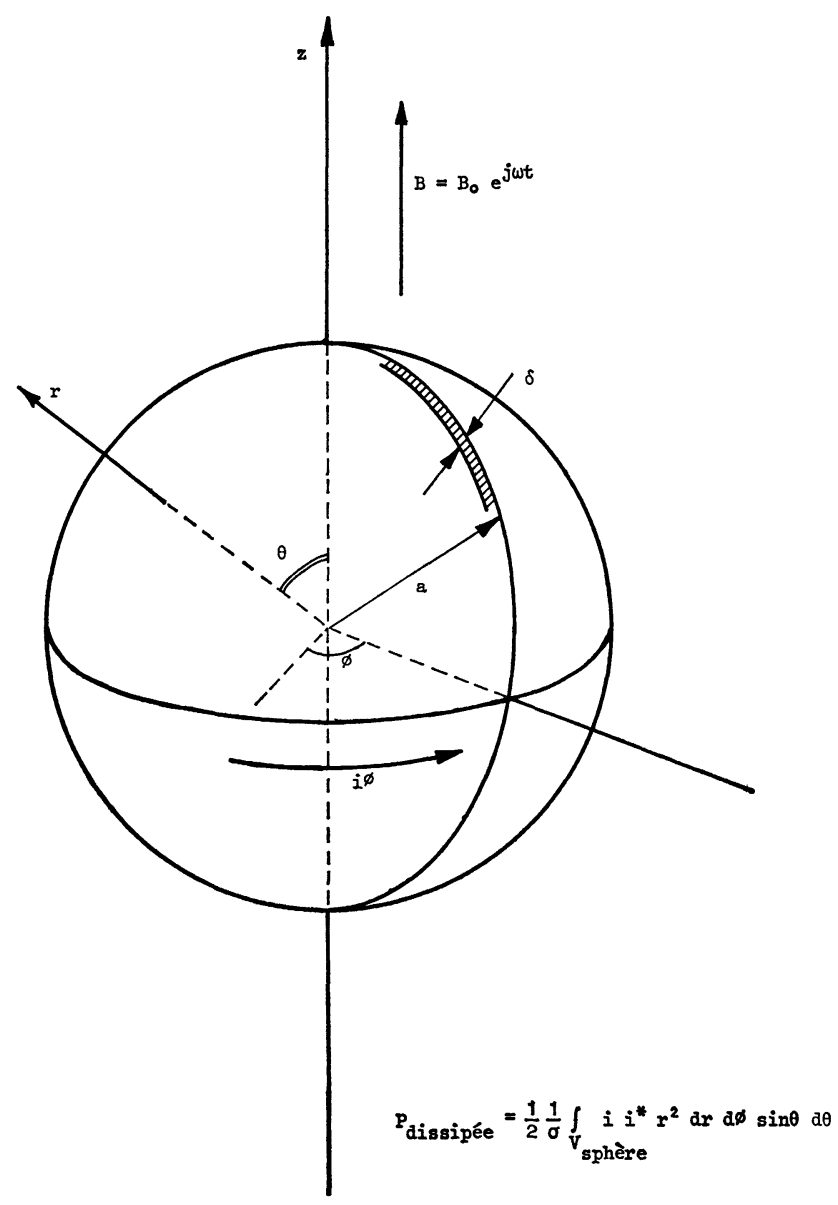

Fig. 1. 
azimutaux et leur densité $i \phi$, assez compliquée dans le cas général, se simplifie dans le cas où l'épaisseur de peau $\delta$ est petite comparée au rayon $a$ de la sphère.

La puissance moyenne dissipée dans la sphère est obtenue à partir de ces courants et a pour expression, dans le cas d'un matériau non magnétique :

$$
\bar{P}=\frac{3 \pi a}{\sigma} \frac{B^{2}}{\mu_{0}^{2}}\left[\gamma \frac{\operatorname{sh} 2 \gamma+\sin 2 \gamma}{\operatorname{ch} 2 \gamma-\cos 2 \gamma}-1\right]
$$

avec :

$a=$ rayon de la sphère,

$$
\gamma=a\left(\frac{\omega \mu_{0} \sigma}{2}\right)^{1 / 2}=\frac{a}{\delta}
$$

$\delta=$ épaisseur de peau,

$\sigma=$ conductivité du matériau.

Dans le cas qui nous intéresse, $\sigma$ est de l'ordre de $10^{4} \mathrm{mho} / \mathrm{m}$ d'où, compte tenu de la fréquence, $\gamma=a / \delta \gg 1$ ( $a$ de l'ordre du $\mathrm{mm})$. La puissance dissipée dans l'échantillon est alors :

$$
\bar{P}=\frac{3 \pi a}{\sigma} H_{0}^{2} \gamma=\frac{3 \pi a^{2} H_{0}^{2}}{\sigma \delta}
$$

Le facteur de qualité $Q_{\text {s }}$ associé à l'échantillon est donné par

$$
\frac{1}{Q_{s}}=\Delta \frac{1}{Q}=\frac{\bar{P}}{\omega W}
$$

avec

$$
W=\frac{\mu_{0}}{2} \int_{\text {cavité }}(H)^{2} \mathrm{~d} v,
$$

énergie stockée dans la cavité.
Afin de simplifier les calculs, introduisons le coefficient :

$$
C_{\mathrm{c}}=\frac{W}{V_{\mathrm{c}} \frac{\mu_{0}}{2} H_{0}^{2}}=\frac{1}{V_{\mathrm{c}}} \int_{\text {cavité }}\left(\frac{H}{H_{0}}\right)^{2} \mathrm{~d} v,
$$

coefficient ne dépendant que du mode et de la géométrie de la cavité. Ce sera donc une constante. Puis :

$$
\frac{1}{K_{m}}=\frac{2}{3} C_{\mathrm{c}}\left(\frac{v_{\mathrm{c}}}{v_{\mathrm{s}}}\right)
$$

$v_{\mathrm{c}}:$ volume de la cavité,

$v_{s}$ : volume de l'échantillon.

Compte tenu de ces modifications, nous obtenons finalement une expression fort simple :

$$
\sigma=\frac{9}{2} \frac{1}{a^{2} \omega\left(\frac{1}{K_{m}} \Delta\left(\frac{1}{Q}\right)\right)^{2}} .
$$

Ayant obtenu l'expression de $\sigma$ en fonction de la perturbation $\Delta(1 / Q)$, il nous faut mesurer cette perturbation d'une manière aussi précise que possible. L'utilisation d'un spectromètre est donc nécessaire.

3. Le spectromètre. - Le montage utilisé consiste à comparer dans un té magique la puissance transmise par la cavité, à la puissance passant par une branche de référence. Cette branche contient un atténuateur et un déphaseur de précision (Fig. 2).

Les mesures consistent à régler l'atténuateur et le déphaseur pour obtenir un zéro sur un détecteur du té magique, ceci à la fréquence de résonance de la cavité.

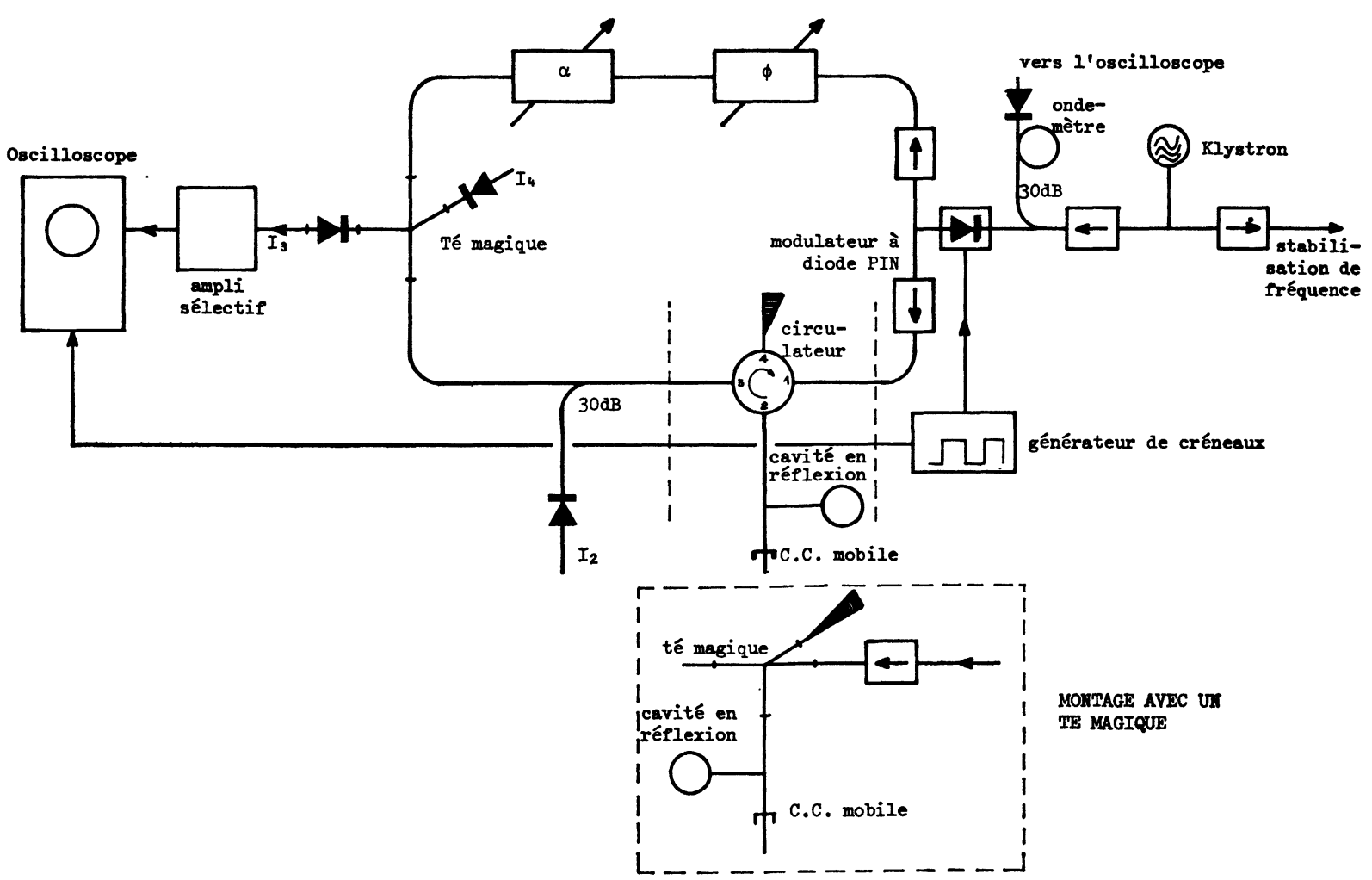

FIG. 2. 
Ce dernier point nécessite une fréquence très stable. Cette stabilisation est obtenue par un ensemble synthétiseur, stabilisation Rhode et Schwarz.

Une mesure est faite sans échantillon et une autre avec échantillon dans la cavité.

Soit $\alpha$ le coefficient de transmission (en champ) de l'atténuateur. Le calcul nous montre que, pour obtenir un zéro dans une des branches du té magique, il faut :

- d'une part, une relation de phase,

- d'autre part,

$$
\alpha_{0}=\frac{1}{2} \frac{\left|1-\beta_{0}\right|}{1+\beta_{0}}
$$

$\beta_{0}$ étant le coefficient de couplage de la cavité.

Lorsqu'on perturbe la cavité, son facteur de qualité $Q_{0}$ devient $Q_{1}$ :

$$
\Delta \frac{1}{Q}=\frac{1}{Q_{1}}-\frac{1}{Q_{0}}
$$

mais :

$$
\beta_{0}=\frac{Q_{0}}{Q_{E}} \quad \text { et } \quad \beta_{1}=\frac{Q_{1}}{Q_{E}} .
$$

$Q_{E}$ est le coefficient de surtension affecté au couplage de la cavité au guide. $Q_{E}$ est une constante pour une géométrie de couplage donnée, d'où :

$$
\frac{1}{\beta_{1}}=\frac{1}{\beta_{0}}+Q_{E} \Delta\left(\frac{1}{Q}\right) \text {. }
$$

Sachant que

$$
\alpha_{1}=\frac{1}{2} \cdot \frac{\left|1-\beta_{1}\right|}{1+\beta},
$$

en remplaçant $\beta_{1}$ par sa valeur :

$$
\begin{array}{rlrl}
Q_{E} \Delta\left(\frac{1}{Q}\right) & =\frac{4\left(\alpha_{1}-\alpha_{0}\right)}{\left(1-2 \alpha_{0}\right)\left(1-2 \alpha_{1}\right)} \text { si } & \beta_{0}<1, \\
& =\frac{4\left(\alpha_{0}-\alpha_{1}\right)}{\left(1+2 \alpha_{0}\right)\left(1+2 \alpha_{1}\right)} & & \beta_{0}>1 .
\end{array}
$$

En fait, nous nous placerons dans le cas le plus favorable : $\beta_{0}=1 \rightarrow\left(\alpha_{0}=0\right)$

$$
Q_{E} \Delta\left(\frac{1}{Q}\right)=\frac{4 \alpha_{1}}{1-2 \alpha_{1}} \text {. }
$$

Un montage identique dans le principe, mais dans lequel nous remplaçons le premier té magique (Fig. 2) par un circulateur, donne une relation un peu différente, due au fait que le té introduit une atténuation de $6 \mathrm{~d} B$.

$$
Q_{E} \Delta\left(\frac{1}{Q}\right)=\frac{2 \alpha_{1}}{1-\alpha_{0}} .
$$

Ce dernier montage n'a pas été retenu lors des mesures en champ magnétique statique, le champ de fuite de l'électro-aimant perturbant le fonctionnement du circulateur à ferrite. Le montage comporte de nombreux isolateurs à ferrite afin d'éviter des interactions entre éléments de circuit.
La source hyperfréquence, un klystron bande $X$ stabilisé en fréquence, est suivie d'un modulateur à diode PIN. Ainsi, nous avons un découpage de l'énergie hyperfréquence permettant un accroissement de sensibilité pour la détection du zéro.

Nous avons fait des mesures à température ordinaire sur des échantillons d'InSb de type $n$ et $p$. Ces échantillons sont sphériques. La précision des mesures est en moyenne de $10 \%$ mais dépend de la cavité utilisée et du volume de l'échantillon. Les mesures sont en accord avec des résultats obtenus par d'autres méthodes. La cavité de mesure est cylindrique et oscille dans le mode $\mathrm{TE}_{114}$.

Les principales difficultés proviennent du positionnement précis de l'échantillon dans la cavité et de son état de surface. Par contre, l'obtention d'un échantillon sphérique, par usure d'un cube ou par électro-érosion, est facile. Ce procédé est donc simple et précis, compte tenu du domaine de mesure. Les résultats sont reproductibles.

4. Conductivité en champ magnétique [7]. - Le milieu étudié ne contient qu'un seul type de porteurs. Etudions la propagation d'une onde électromagnétique dans un solide conducteur soumis à un champ magnétique. Ce milieu de constante diélectrique statique relative $K$, de perméabilité relative $\mu_{r}=1$, contient $N$ électrons libres par unité de volume. Il est soumis à une induction magnétique $B_{0}$. Nous supposerons par ailleurs que les électrons ont une masse effective isotrope $m^{*}$.

Les équations de Maxwell et les équations caractéristiques du milieu que sont les équations de transport, vont nous permettre d'écrire des relations entre le champ électrique $\mathbf{E}$ et le courant $\mathbf{J}$ :

$$
\mathbf{J}=\overrightarrow{\boldsymbol{\sigma}} \mathbf{E}
$$

où $\vec{\sigma}$ est le tenseur conductivité. Dans le cas où $B_{0}$ est dirigé normalement au front de l'onde, ce tenseur s'écrit :

$$
\overline{\bar{\sigma}}=\sigma_{0}\left[\begin{array}{ccc}
\frac{1+j \omega \tau}{D} & j \frac{\omega_{L} \tau}{D} & 0 \\
-j \frac{\omega_{L} \tau}{D} & \frac{1+j \omega \tau}{D} & 0 \\
0 & 0 & \frac{1}{1+j \omega \tau}
\end{array}\right]
$$

avec

$$
\begin{gathered}
D=(1+j \omega \tau)^{2}+\omega_{L}^{2} \tau^{2} \\
\sigma_{0}=\frac{N e^{2} \tau}{m^{*}}
\end{gathered}
$$

$\tau:$ temps de relaxation des électrons, $\omega_{L}=\mathrm{e} B / m^{*}:$ pulsation cyclotronique,

$\omega$ : pulsation de l'onde.

Pour l'antimoniure d'indium, $\tau \simeq 10^{-12} \mathrm{~s}$, d'où pour 
des mesures en bande $X: \omega \tau \ll 1$. Le tenseur va donc se simplifier :

$$
\sigma=\sigma_{0}\left[\begin{array}{ccc}
\frac{1}{1+\omega_{L}^{2} \tau^{2}} & j \frac{\omega_{L} \tau}{1+\omega_{L}^{2} \tau^{2}} & 0 \\
-j \frac{\omega_{L} \tau}{1+\omega_{L}^{2} \tau^{2}} & \frac{1}{1+\omega_{L}^{2} \tau^{2}} & 0 \\
0 & 0 & 1
\end{array}\right]
$$

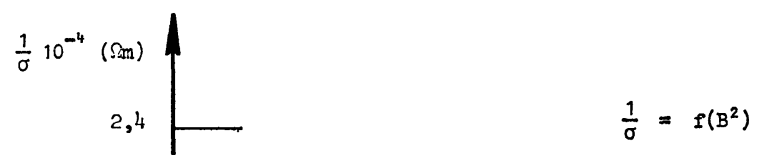

L'appareillage précédent muni d'une cavité en polarisation circulaire doit nous permettre de mesurer les éléments du tenseur, en nous inspirant d'une méthode analogue à celle utilisée pour les ferrites. L'anisotropie en champ magnétique ainsi que la forte conductivité se traduisent par une épaisseur de peau non constante. Les pertes dans l'échantillon sont donc difficilement calculables. Des calculs sont en cours pour effectuer le lien entre la perturbation du mode dans la cavité et les paramètres du matériau. Nous avons effectué des

InSb type $\mathrm{n}$

$\mathrm{T}=300^{\circ} \mathrm{K}$

2,

, 0

2,2

-

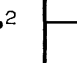

1,8



1,6

1,4

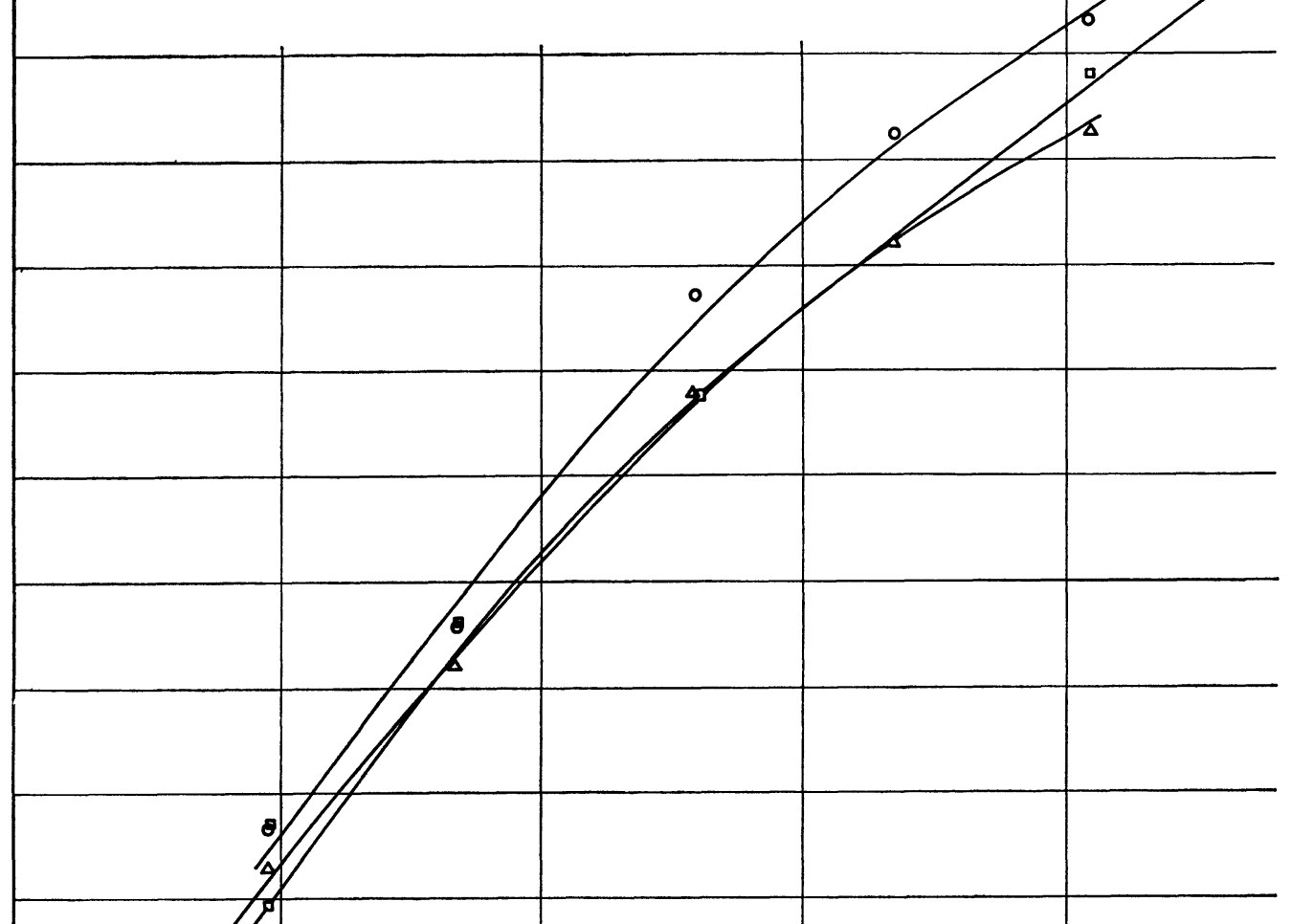

1,

西



1,0
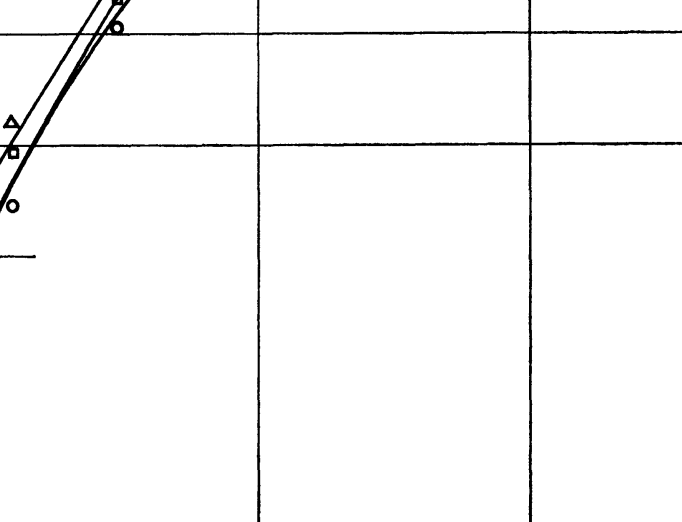

$2 \frac{1}{10} 20$

FIG. 3. 


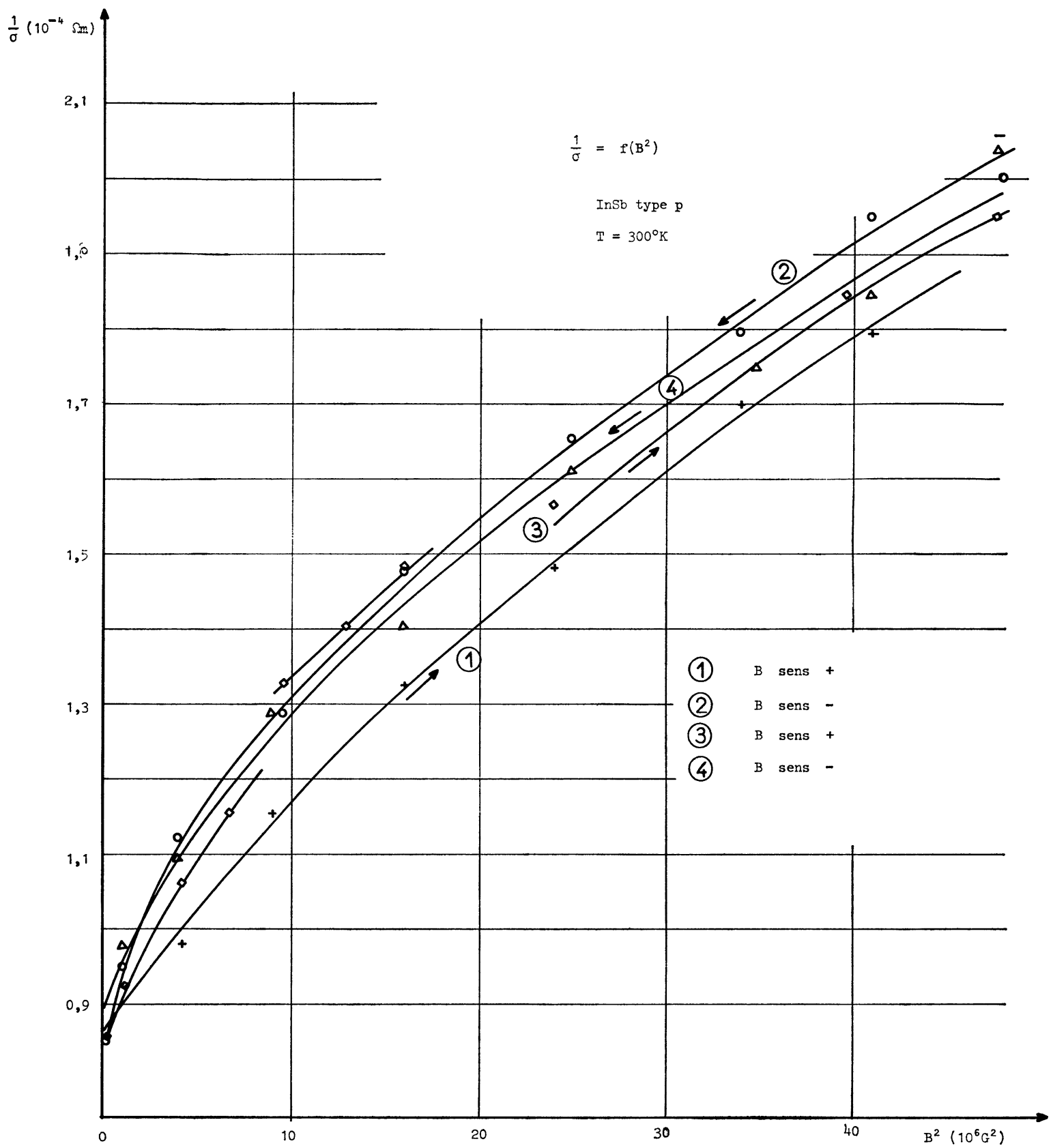

FIG. 4.

mesures de $\sigma$ en champ magnétique statique ; sans nous préoccuper de la forme tensorielle, nous obtenons les courbes des figures 3 et 4 .
Des mesures vont également être faites à $T=77^{\circ} \mathrm{K}$. A cette température, le caractère anisotropique est encore plus marqué.

\section{Bibliographie}

[1] Allerton (G. L.), Seiferty (J. R.), Resistivity measurements of semiconductors at nine thousand megacycles, I. R. E. Trans., 1960, I 9, 1, 175-179.

[2] Heaton (A. G.), Determination of semiconductor resistivity by microwave measurements, Proc. I. E. E., 1968, 115, 5, 742-746.

[3] Jacobs (H.), Brand (F. A.), Meindl (J. D.), Benanti (M.), BENJAMIN (R.), Electrodeless measurement of semiconductor resistivity at microwave frequencies, Proc. I. R. E., 1961, 49, 928-932.

[4] Champlin, Holm (J. D.), Glover (G. H.), Electrodeless determination of semiconductor conductivity from $\mathrm{TE}_{01}$ mode reflectivity, J. Appl. Phys., janvier 1967, 38, 96-98.

[5] Champlin, Holm (J. D.), Glover (G. H.), Bulk microwave conductivity of semiconductors determined from $T E_{01}^{0}$ mode reflectivity of boule surface, I. E. E. E. Trans., 1969, IM 18, 2, 105.

[6] SMYthe, Static and dynamic electricity, Ed. McGraw Hill, 396-400, seconde édition, 1950.

[7] Veilex (R.), Thèse de Doctorat ès sciences physiques, Faculté des Sciences de l'Université de Paris, 1964. 